\title{
Qualidade de MEDIÇões e NeUTRALIZAÇÃo de EFLUENTES ALCALINOS COM DIÓXIDO DE CARBONO
}

\section{QUALITY OF MEASUREMENTS AND NEUTRALIZATION OF ALKALINE EFFLUENTS WITH CARBON DIOXIDE}

\section{ELOY ALVES FERREIRA FILHO}

Mestre em Engenharia e Ciência dos Materiais. Programa de Pós-Graduação em Engenharia e Ciência dos Materiais PPGECM. Universidade São Francisco - USF

\section{QueENIE SIU HANG CHUI}

Doutora em Química Analítica Ambiental. Docente do PPGECM - USF

Recebido: 06/09/05 Aceito: 02/05/06

\section{RESUMO}

$\mathrm{O}$ controle de $\mathrm{pH}$ de efluentes industriais, para fins de descarga, é estabelecido por leis estaduais e federais. Devido ao não conhecimento da incerteza de suas mediçôes e principalmente não se conhecendo sua rastreabilidade a padrôes nacionais e/ou internacionais, pode ocorrer não conformidade ao se permitir a descarga de efluente ao meio ambiente. Este trabalho enfoca a qualidade das medições de $\mathrm{pH}$, através dos aspectos da rastreabilidade e da incerteza de medição, seguindo os conceitos introduzidos pela ISO GUM. O experimento foi realizado com amostra de efluente alcalino coletado de uma indústria têxtil, envolvendo a reação de neutralização usando o dióxido de carbono. Resultados de $\mathrm{pH}$ acompanhados de suas incertezas oferecem informação mais completa na prevenção contra descargas impróprias de efluentes ao meio.

PALAVRAS-CHAVE: Efluente alcalino, dióxido de carbono, $\mathrm{pH}$, incerteza

\begin{abstract}
State and federal laws rule the control of $p H$ of industrial effluents aiming discharge and environmental protection. Improper effluent discharges can occur if obtained $\mathrm{pH}$ measurements do not meet traceability to national andlor international standards and are not associated with their uncertainties. This work focuses the quality of the $\mathrm{pH}$ measurements and follows the ISO GUM instructions by demonstrating the traceability and estimating the uncertainty of the measurements. The experiment was carried out using an alkaline effluent sample of a textile industry, involving the neutralization reaction with carbon dioxide. Having $p H$ measurements associated with its uncertainty can prevent risks of improper discharge of effluent to the environment.
\end{abstract}

KEYWORDS: Alkaline effluent, carbon dioxide, $p H$, uncertainty

\section{INTRODUÇÃO}

A grande preocupação com o controle do meio ambiente tem motivado as indústrias e órgãos ambientais do governo a minimizar os efeitos prejudiciais da poluição ambiental. A manutenção dos recursos hídricos, tanto para o consumo industrial como doméstico, obriga o controle de parâmetros previamente estabelecidos, de modo a permitir a preservação da fauna e flora, além do reaproveitamento das águas dos mananciais.

O Conselho Nacional do Meio Ambiente - CONAMA, órgão consultivo e deliberativo do Sistema Nacional do Meio Ambiente - SISNAMA, foi instituído pela Lei 6938/81, que dispõe sobre a Política Nacional do Meio Ambiente, regulamentada pelo Decreto 99274/90. A Resolução no 20 do CONAMA, de 18.06.86, estabeleceu nove classes de rios em função dos usos preponderantes para águas doces, salinas e salobras do Território Nacional. Diversas leis federais foram estabelecidas integrando a Política Nacional do Meio Ambiente, porém a grande evolução veio com a lei no 9433 , de 08.01.97, que instituiu a Política e o Sistema Nacional de Gerenciamento de Recursos Hídricos, em que cabe a cada Estado do Território Nacional instituir o Sistema Integrado de Gerenciamento de Recursos Hídricos, que prevê congregar os órgãos estaduais e municipais e a sociedade civil, e assegurar meios financeiros e institucionais. $\mathrm{Na}$ Constituição do Estado de São Paulo, o artigo 208 veda o lançamento de efluentes industriais e esgotos urbanos sem o devido tratamento em qualquer corpo de água. Para estabelecer limites e condiçôes para os corpos de água em função dos usos e o sistema de licenciamento para as fontes de poluição foi criado o Decreto no 8468 , de 08.09.79, que regulamentou a Lei Estadual de 31.05.76.

No que tange ao $\mathrm{pH}$, o artigo de no 18 desta lei estabelece que "os efluentes de qualquer fonte poluidora somente poderão ser lançados, direta ou indiretamente, nas coleções de água, desde que os valores se encontrem entre 5,0 (cinco inteiros) e 9,0 (nove inteiros)"; por outro lado, o artigo de no 19 indica que "os efluentes somente poderão ser lançados em sistema de esgotos, provido de tratamento com capacidade e de tipo adequados, conforme previsto no $\int 4^{\circ}$ deste artigo, se obedecerem às condições de $\mathrm{pH}$ entre 6,0 (seis inteiros) e 10,0 (dez inteiros)". 
A condição de $\mathrm{pH}$ dos efluentes decorre da contaminação provocada pelos diversos processos industriais de fabricação. O contato da água com substâncias como soda cáustica, potassa cáustica, cal, entre outras, libera um efluente final alcalino altamente prejudicial aos mananciais, necessitando de uma solução corretiva para redução do $\mathrm{pH}$ para níveis toleráveis. Em geral empregam-se os ácidos inorgânicos, sulfúrico e clorídrico, para efluentes provenientes de indústrias muitas vezes localizadas em áreas de mananciais. Os danos causados por estes ácidos agressivos e os altos riscos advindos de sua utilização e problemas durante o transporte podem comprometer seriamente o meio ambiente.

A alternativa usando o dióxido de carbono como neutralizador de efluentes alcalinos é justificada pela sua facilidade operacional. Além de evitar os riscos provenientes de vapores tóxicos, queimaduras e outras lesões, que ocorrem com a manipulação de ácidos inorgânicos, reduz os custos de manutenção devido ao seu baixo potencial de corrosão. Devido às características do sistema tampão $\mathrm{CO}_{3}{ }^{2-} / \mathrm{HCO}_{3}$ - presente, o pH final de neutralização do efluente alcalino não se altera mesmo com a adição de excesso de dióxido de carbono. Isto representa uma vantagem sobre o processo com ácidos minerais inorgânicos, pois um pequeno excesso destes já levaria o $\mathrm{pH}$ do efluente a valores mais baixos, levando a conseqüências adversas ao meio ambiente.

$\mathrm{O}$ controle de $\mathrm{pH}$ dos processos de neutralização é simples, porém observase que, em geral, não existe rastreabilidade para suas mediçōes. Apesar de as empresas dedicarem grandes esforços na obtenção de certificados de sistemas de gestão ambiental, seus laboratórios muitas vezes não têm o controle de calibração de seus equipamentos validado, não demonstram a rastreabilidade de soluções tampão a padrões nacionais e/ou internacionais, não atendem aos procedimentos adequados de limpeza e manutenção de eletrodos, enfim não demonstram efetivamente a confiabilidade metrológica de suas mediçôes.

Muitas vezes as medições de $\mathrm{pH}$ obtidas, utilizando equipamentos sem a devida calibração e controle (Fraga et al, 2002), podem estar indicando valores permitindo o descarte de efluente em $\mathrm{pH}$ dentro do padrão ambiental estabelecido ( $\mathrm{pH}$ entre 5,0 a 9,0 conforme atendendo ao artigo 18 ou $\mathrm{pH}$ entre 6,0 a 10,0 com respeito ao artigo 19); porém, na realidade, pode estar ocorrendo não conformidade, devido ao não conhecimento da incerteza desses valores. E a incerteza somente pode ser estabelecida quando se conhece a rastreabilidade das medições (Oliveira, 2003).

Com referências às normas da série NBR/ISO 14000 para a gestão ambiental, esta compartilha princípios comuns com as normas da série NBR/ISO 9000 relativas ao sistema da qualidade. No entanto em auditorias de sistemas de gestão ambiental, pouca ênfase é dada à confiabilidade em resultados de medições gerados nos laboratórios das empresas.

Este trabalho enfoca a qualidade das medições de $\mathrm{pH}$, através da abordagem da rastreabilidade e incerteza da medição, em um estudo de caso envolvendo o processo alternativo de neutralização de efluente alcalino usando o dióxido de carbono.

\section{RASTREABILIDADE E INCERTEZA DE MEDIÇÖES}

Toda medição possui erros de natureza sistemática ou aleatória, o que acrescenta uma incerteza ao resultado. Resultados de mediçōes expressos apenas como médias sem as respectivas incertezas carecem de significado, pois não dão a informação completa sobre a medição. Assim, não podem ser comparados com os valores designados como limites e/ou valores de referência. Para conferir qualidade às mediçōes é necessário conhecer a rastreabilidade (VIM, 1995) e a incerteza associada aos seus valores.

A rastreabilidade das mediçôes tornou-se obrigatória (CNI, 2005), ou seja, qualquer resultado de medição deve fazer parte de uma cadeia contínua de valores, denominada "cadeia de rastreabilidade” (ISO GUM, 1998). Em todos os níveis dessa cadeia, deve-se adotar o Sistema Internacional de Unidades - SI (BIPM, 1998), bem como deve ser feita uma investigação sobre a incerteza da medição. Em todos os países, os Institutos Nacionais de Metrologia têm o papel de controlar e manter os valores das propriedades específicas atribuídas aos padróes nacionais. No Brasil, esse papel é desempenhado pelo Instituto Nacional de Metrologia, Normalização e Qualidade Industrial - INMETRO.
A incerteza total do resultado de uma medição resulta da soma das incertezas que se originam de diversos fatores de influência do processo de medição e que geram variabilidades no cômputo do resultado final. A incerteza se propaga desde a calibração da grandeza primária até a utilização final do instrumento, incorporando as incertezas dos vários laboratórios hierarquicamente organizados, que tenham participado do processo de medição. Apesar de a incerteza refletir principalmente características de padrões e métodos, ela contém também componentes do próprio instrumento calibrado.

A forma de expressar a incerteza da medição deve ser padronizada para que todos os laboratórios se expressem da mesma maneira, e para que a propagação dessa incerteza se dê de forma conhecida. De acordo com as instruções do Guia para a Expressão da Incerteza de Medição (ISO GUM, 1998), o técnico deve calcular e avaliar a incerteza padrão tipo $\mathrm{A}, \mathrm{a}(\mathrm{s})$ incerteza(s) padrão tipo $\mathrm{B}$, a incerteza combinada e a incerteza expandida, que é a sua expressão final. Por definição, a incerteza padrão tipo A $\left(\mu_{A}\right)$ é aquela obtida por meios puramente estatísticos, ou seja, é avaliada a partir da análise de uma série de observações, calculando-se o desvio padrão da média seguindo os métodos da estatística clássica. A incerteza padrão tipo $\mathrm{B}\left(\mu_{B}\right)$ também deve ser dada na forma de desvio padrão, porém seguindo o princípio da estatística bayesiana (Harry e Waller, 1994), ou seja, consiste em admitir que os possíveis valores das quantidades em estudo seguem uma distribuição de probabilidades que esteja de acordo com todo o conhecimento e informaçôes disponíveis sobre as variabilidades dessas quantidades (Vuolo, 1999); dentre elas incluem-se as informações técnicas fornecidas por fabricantes dos instrumentos utilizados, a incerteza de padrôes utilizados para a devida calibração do instrumento (evidenciada no seu relatório de calibração), a incerteza observacional (paralaxe, interpolação, instabilidade da indicação digital, etc.), as incertezas ambientais, que compreendem as influências devidas à temperatura ambiente e outras de importância para cada situação específica em estudo. Algumas das distribuiçôes comumente consideradas são a distribuição retangular, a distribuição triangular e a de Laplace-Gauss (Harry e Waller, 1994). A combinação 
de incertezas padrão tipo A e tipo B deverá ser feita pela fórmula usual de propagação de incertezas, para chegar à incerteza expandida $(U)$, que expressa a incerteza combinada multiplicada por um fator de abrangência, $K$, obtido em função do nível de confiança e número de graus de liberdade efetivo, usando a fórmula de Welch-Satterthwaite (ISO GUM, 1998; Vuolo, 1999).

\section{A RASTREABILIDADE NA MEDIÇÃO DE PH}

$\mathrm{Na}$ rotina de laboratórios comuns, as mediçôes são obtidas utilizando o medidor de $\mathrm{pH}$ e dois eletrodos, o indicador (geralmente o de vidro) e o de referência (de calomelano saturado ou de prata-cloreto de prata). O sistema medidor e eletrodos é previamente calibrado com uma ou mais soluçôes tampão de concentração hidrogeniônica conhecida, adquiridas no mercado. Muitas dúvidas são levantadas a respeito da rastreabilidade dos produtos comercializados, uma vez que o adequado preparo dessas soluçóes requer a utilização de materiais de referência primários (Buck et al, 2002), que são sais de pureza e de estabilidade comprovadas. Para a certificação destes materiais, suas medições devem estar referidas a sistemas primários para a medição de $\mathrm{pH}$, cujas operaçôes são atribuídas aos laboratórios nacionais metrológicos (Koch, 1997; Baucke et al, 1998). Até o momento, materiais de referência para fornecer rastreabilidade e confiabilidade às medições de $\mathrm{pH}$ não são produzidos no Brasil. Porém o INMETRO, através de sua Diretoria de Metrologia Científica e Industrial, cumprindo um de seus papéis de Instituto Nacional de Metrologia do País, implantou na sua Divisão de Metrologia Química o sistema primário para a medição de pH (Souza, 2003) com o objetivo de possibilitar a certificação de material de referência para soluções de $\mathrm{pH}$. As soluções tampão assim certificadas fornecerão rastreabilidade e confiabilidade aos padrōes secundários e medições de $\mathrm{pH}$ aos usuários.

\section{MATERIAL E MÉTODO}

Considerando o processo de neutralização usando dióxido de carbono, foi montado um protótipo similar ao existente em uma estação de tratamento de efluentes, usando uma bomba de circulação e um sistema de injeção de $\mathrm{CO}_{2}$ com venturi e plug poroso, para tornar as bolhas pequenas (Figura 1). $\mathrm{O}$ plug poroso era conectado a um cilindro de $8 \mathrm{~kg}$ de $\mathrm{CO}_{2}$ tipo post-mix, com regulador de pressão de duplo estágio marca White Martins tipo R-201 e fluxômetro de $0-15 \mathrm{l} / \mathrm{min}$ calibrado para uma pressão de $3,5 \mathrm{kgf} / \mathrm{cm}^{2}$ a fim de controlar a injeção de $\mathrm{CO}_{2}$.

Uma cuba em acrílico de $20 \mathrm{~cm}$ de largura, $60 \mathrm{~cm}$ de comprimento e $20 \mathrm{~cm}$ de profundidade, com capacidade de 24 litros foi usada para conter o efluente. O volume de aproximadamente 18 litros de efluente utilizado no experimento alcançava até $15 \mathrm{~cm}$ de altura.

Mangueiras poli-flow foram utilizadas para interligar a saída do fluxômetro à entrada da válvula de retenção e mangueira incolor de $12,5 \mathrm{~mm}$, interligando a saída da cuba na entrada da bomba, saída da bomba, entrada no injetor de $\mathrm{CO}_{2}$ e saída do injetor na entrada da cuba.

Complementando o sistema, foram usados uma válvula de retenção Danfoss diâmetro 3/8 de polegadas, um injetor com sinterizado cerâmico e uma bomba de recirculação marca DANCOR com entrada e saída de 3/4 de polegadas, de recirculação com motor de $1 / 8 \mathrm{CV}$ e $110 \mathrm{~V}$.

$\mathrm{O}$ medidor de $\mathrm{pH}$ marca Orion modelo 520 A foi usado para o controle do processo. Para a calibra-

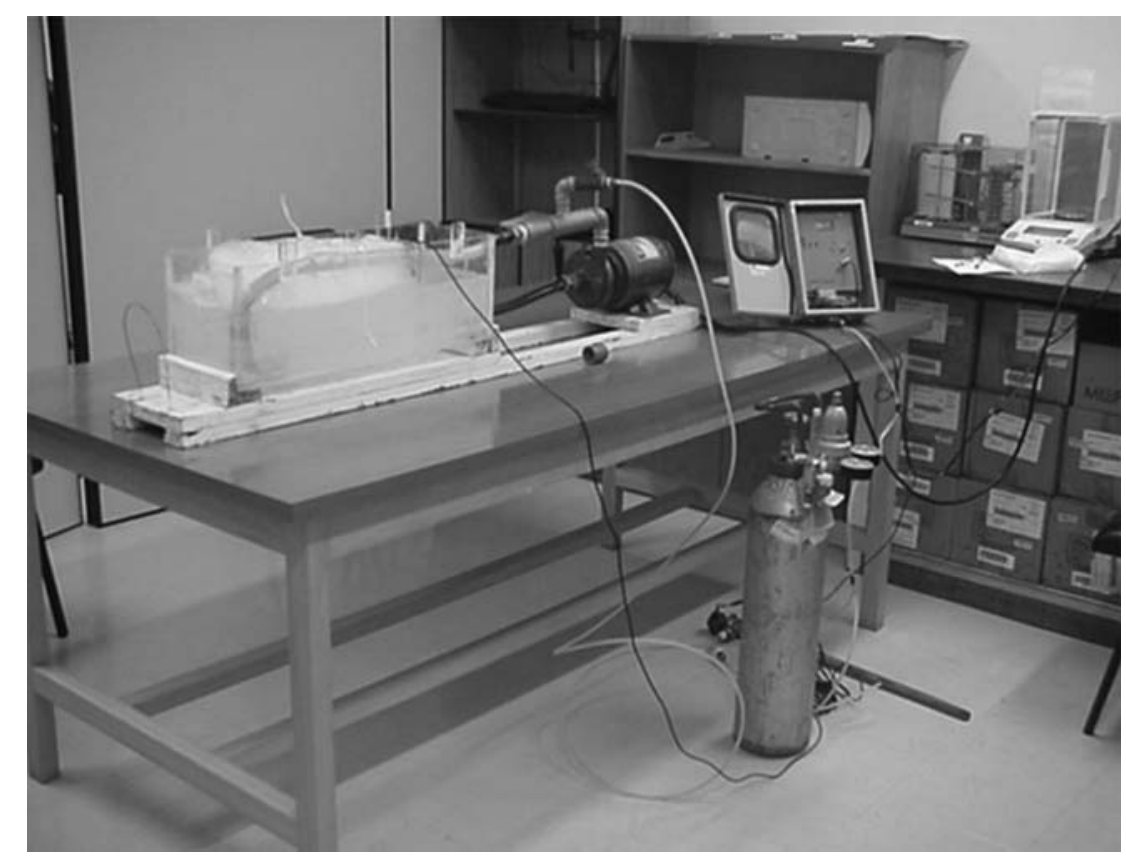

Figura I - Protótipo montado para o estudo de neutralização de
efluente alcalino usando dióxido de carbono ção do medidor, foram usadas 2 soluçóes tampão, de $(7,00 \pm 0,01)$ e $(10,01 \pm 0,02)$ unidades de $\mathrm{pH}$, materiais de referência certificados por laboratório do National Institute of Standards and Technology - NIST (EUA).

Para a verificação das condições operacionais do protótipo proposto, foram preparadas soluções aquosas de hidróxido de amônio com $\mathrm{pH}$ de aproximadamente 12 , adicionando, a um volume de 18 litros de água de torneira, alíquotas de $10 \mathrm{ml}$ de reagente $\mathrm{NH}_{4} \mathrm{OH} 28 \%$, até que o $\mathrm{pH}$ da solução resultasse em torno de 12 . Em seguida, o processo de neutralização com dióxido de carbono foi simulado utilizando uma amostra de 18 litros de efluente alcalino coletado na Estação de Tratamento de Efluentes da Empresa Vicunha Textil Ltda, Unidade I em Itatiba, São Paulo, em 19/09/2003 às 18:00 horas. $\mathrm{O}$ efluente com vazão de $42 \mathrm{~m}^{3} / \mathrm{h}$ na entrada da estação apresentava os seguintes parâmetros: pH 11; sólidos totais dissolvidos de $210 \mathrm{mg} / \mathrm{l}$; demanda química de oxigênio (DQO) expresso como $766 \mathrm{mg} / \mathrm{l} \mathrm{de}_{2}$ e temperatura de $61{ }^{\circ} \mathrm{C}$.

\section{RESULTADOS E DISCUSSÂO}

Os ensaios preliminares de neutralização utilizando 18 litros de soluções 
aquosas de hidróxido de amônio de $\mathrm{pH}$ de aproximadamente 12 foram repetidos 16 vezes, para observar a repetitividade do experimento. Os resultados estão mostrados na Tabela 1.

Pode-se observar que os ensaios apresentaram boa repetitividade, tendo sido consumidos 4,28 litros (valor médio) de $\mathrm{CO}_{2}$, com desvio padrão de 0,49 litros, representando um coeficiente de variação $(\mathrm{CV})$ de $11 \%$. O $\mathrm{pH}$ inicial médio de 12,53 com desvio padrão de $0,57(\mathrm{CV}=4,5 \%)$ foi reduzido a um $\mathrm{pH}$ final com valor médio de 6,59 e desvio padrão de $0,14(\mathrm{CV}=2,1 \%)$. Em seguida foram efetuados os cálculos para obter a incerteza final da medição de $\mathrm{pH}$, seguindo as instruções do ISO GUM.

- Cálculo da incerteza padrão tipo $\mathrm{A}, u_{A}$, com base na dispersão das medições em replicatas em que $S_{(\mathrm{pH})}$ é o desvio padrão dos 16 resultados de $\mathrm{pH}$ e $\mathrm{n}=16$ é o número de replicatas efetuadas.

$\mathrm{u}_{\mathrm{A}}=\mathrm{S}_{(\mathrm{pH})} / \mathrm{n}^{1 / 2}$

$\mathrm{u}_{\mathrm{A}}=0,14 / 16^{1 / 2}=0,035$

- Cálculo da incerteza padrão do tipo $\mathrm{B}, u_{B}$, utilizando as informaçôes técnicas (exatidão do instrumento) for- necidas pelo fabricante do instrumento, em que I é o valor da incerteza $( \pm 0,01)$ declarada pelo fabricante do instrumento dividido por fator $\left(3^{1 / 2}\right)$, consideran-

$\mathrm{u}_{\mathrm{B}}=\mathrm{I} / 3^{1 / 2}$

$u_{B}=0,01 / 3^{1 / 2}=0,0058$

do a distribuição retangular.

- Cálculo da incerteza padrão do tipo $\mathrm{B}, u_{B}$, considerando as informações técnicas sobre a resolução $(\mathrm{R})$ do instrumento, sendo que sua incerteza é estimada como a metade do valor de sua resolução. O ISO GUM sugere, para resolução de indicadores digitais, considerar a distribuição retangular, portanto o valor da incerteza é dividido

$\mathrm{u}_{\mathrm{B}}=(\mathrm{R} / 2) / 3^{1 / 2}$

$\mathrm{u}_{\mathrm{B}}=(0,01 / 2) / 3^{1 / 2}=0,0029$

por raiz quadrada de 3 .

- Cálculo da incerteza padrão do tipo $\mathrm{B}, u_{B}$, levando-se em conta a incerteza dos materiais de referência NIST usados para a calibração do medidor de $\mathrm{pH}$. Considerando que a incerteza declarada compreendia o intervalo de 95\% de confiança de uma distribuição normal, seu valor é dividido por 2 .
$\mathrm{u}_{\mathrm{B}}(\mathrm{pH} 7)=\mathrm{U}_{\mathrm{MRC7}} / 2$

$\mathrm{u}_{\mathrm{B}}(\mathrm{pH} 7)=0,01 / 2=0,005$

$\mathrm{u}_{\mathrm{B}}(\mathrm{pH} 10)=\mathrm{U}_{\mathrm{MRC} 10} / 2$

$\mathrm{u}_{\mathrm{B}}(\mathrm{pH} 10)=0,02 / 2=0,01$

- Cálculo da incerteza combinada $\left(\mathrm{u}_{\mathrm{C}}\right)$, que resulta da raiz quadrada da soma quadrática das várias parcelas, que representam as incertezas tipo $\mathrm{A}$ e tipo B.

$$
\begin{aligned}
& \mathrm{u}_{\mathrm{C}}^{2}=\mathrm{u}_{\mathrm{A}}^{2}+\mathrm{u}_{\mathrm{B}(\text { instr })}^{2}+\mathrm{u}_{\mathrm{B}(\text { resol })}^{2}+ \\
& +\mathrm{u}_{\mathrm{B}(\mathrm{pH} 7)}^{2}+\mathrm{u}_{\mathrm{B}(\mathrm{pH} 10)}^{2}
\end{aligned}
$$

$\mathrm{u}_{\mathrm{C}}^{2}=0,035^{2}+0,0058^{2}+0,0029^{2}+$

$+0,005^{2}+0,01^{2}=0,0363$

- Cálculo dos graus de liberdade efetivos $\left(v_{\text {ef }}\right)$ usando a expressão de Welch-Satterthwaite (ISO GUM, 1998; Vuolo, 1999).

$$
v_{\text {ef }}=\frac{\left(\mathrm{u}_{\mathrm{C}}\right)^{4}}{\left(\mathrm{u}_{\mathrm{A}}\right)^{4} /(\mathrm{n}-1)}=\frac{0,0363^{4}}{0,035^{4} / 15}=20
$$

Tabela I - Resultados do experimento de neutralização de solução de $\mathrm{NH}_{4} \mathrm{OH}$ utilizando $\mathrm{CO}_{2}$ gasoso

\begin{tabular}{ccccc}
\hline Data & pH inicial & pH final & Tempo (s) & $\begin{array}{c}\text { Quantidade CO }_{2} \\
\text { consumida (litros) }\end{array}$ \\
\hline $8 / 8 / 2003$ & 12,5 & & 18 & 3,6 \\
$8 / 8 / 2003$ & 12,4 & 6,6 & 20 & 4,0 \\
$8 / 8 / 2003$ & 12,6 & 6,5 & 21 & 4,2 \\
$15 / 8 / 2003$ & 12,3 & 6,7 & 20 & 4,0 \\
$15 / 8 / 2003$ & 13,6 & 6,5 & 26 & 5,2 \\
$22 / 8 / 2003$ & 12,5 & 6,8 & 20 & 4,0 \\
$22 / 8 / 2003$ & 13,2 & 6,7 & 24 & 4,8 \\
$12 / 9 / 2003$ & 12,0 & 6,5 & 20 & 4,0 \\
$19 / 9 / 2003$ & 13,2 & 6,8 & 25 & 5,0 \\
$10 / 10 / 2003$ & 12,5 & 6,4 & 20 & 4,0 \\
$10 / 10 / 2003$ & 12,3 & 6,6 & 22 & 4,4 \\
$17 / 10 / 2003$ & 12,3 & 6,4 & 20 & 4,0 \\
$17 / 10 / 2003$ & 12,6 & 6,5 & 21 & 4,0 \\
$17 / 10 / 2003$ & 12,6 & 6,7 & 25 & 5,2 \\
$24 / 10 / 2003$ & 12,4 & 6,8 & 18 & 5,0 \\
$31 / 10 / 2003$ & 12,8 & 6,5 & 22 & 3,6 \\
\hline
\end{tabular}


Utilizando a tabela de T-Student, para graus de liberdade 20 , o fator de abrangência é de 2,09 (95\% de confiança), que é usada para o cálculo da incerteza expandida.

- Cálculo da incerteza expandida $(U)$ para o valor de $\mathrm{pH}$ final na neutralização de soluções aquosas de $\mathrm{NH}_{4} \mathrm{OH}$.

$\mathrm{U}=\mathrm{u}_{\mathrm{C}} \times 2,09$

$\mathrm{U}=0,0363 \times 2,09=0,076$

Como resultado final para o valor de $\mathrm{pH}$ e sua incerteza tem-se $(6,59 \pm 0,08)$.

Após ter sido verificado que a reação de neutralização acontecia sem problemas operacionais, foi conduzido o experimento usando efluente coletado da Estação de Tratamento de Efluentes da Vicunha Têxtil. Uma amostra de 18 litros desse efluente foi despejada na cuba de acrílico, após o que foi introduzido um fluxo de $\mathrm{CO}_{2}$ de 3 litros $\left(0,55 \mathrm{~m}^{3} \mathrm{~kg}^{-1}\right)$. A solução de $\mathrm{pH}$ inicial 11 foi neutralizada após 15 segundos, tendo o $\mathrm{pH}$ sido reduzido para 6,50. Considerando medição sem repetição e utilizando os valores obtidos para as incertezas do tipo B no caso acima, foi estimada a incerteza do valor de $\mathrm{pH}$.

- Cálculo da incerteza combinada $\left(u_{C}\right)$ para o valor de $\mathrm{pH}$ resultante da neutralização de amostra de efluente de indústria têxtil:

$\mathrm{u}_{\mathrm{C}}^{2}=\mathrm{u}_{\mathrm{B}(\mathrm{instr})}^{2}+\mathrm{u}_{\mathrm{B}(\mathrm{resol})}^{2}+\mathrm{u}_{\mathrm{B}(\mathrm{pH} 7)}^{2}+$

$+\mathrm{u}_{\mathrm{B}}^{2}(\mathrm{pH} 10)$

$\mathrm{u}_{\mathrm{C}}^{2}=0,0058^{2}+0,0029^{2}+0,005^{2}+$

$+0,01^{2}=0,013$

- Cálculo da incerteza expandida $(U)$ para o valor de $\mathrm{pH}$ final na neutralização de amostra de efluente de indústria têxtil. Considerando o nível de confiança de 95\%, 1,96 corresponde ao fator de abrangência, K, que é multiplicado pelo valor obtido para a incerteza combinada.

$\mathrm{U}=1,96 \times 0,013=0,025$

$\mathrm{O}$ valor final de $\mathrm{pH}$ foi expresso como $(6,50 \pm 0,03)$. Portanto, pode-se dizer que, no final da reação, com $95 \%$ de confiança, o valor de $\mathrm{pH}$ se encontrava entre os limites de 6,47 a 6,53.

\section{CONCLUSÃO}

Pode-se observar que o resultado final de $\mathrm{pH}$ com a incerteza associada indicou valores que confirmam existirem condiçóes de controle para fins de descarga, quando se consideram os limites admissíveis de $\mathrm{pH}$ 6,0 a 10,0 para a região onde a estação de tratamento está localizada.

Reações de neutralização de efluentes alcalinos, contando com medições de $\mathrm{pH}$ com rastreabilidade conhecida, representam uma segurança para evitar riscos de despejo de efluentes com $\mathrm{pH}$ fora dos níveis indicados pelas regras ambientais vigentes. $\mathrm{O}$ desconhecimento da incerteza associada à medição de $\mathrm{pH}$ possibilita que o responsável pelo controle de descarga do efluente tome decisões erradas.

Os procedimentos para a avaliação de incerteza tipo A usam métodos estatísticos bem conhecidos. No entanto não existe procedimento detalhado para a avaliação das incertezas tipo B. O avaliador deve escolher uma distribuição de probabilidades para valores possíveis da grandeza, sendo que essa escolha deve considerar as informações disponíveis sobre o mensurando e a medição. As instruçôes recomendadas pelo ISO GUM têm tido ampla repercussão e aceitação pelas instituiçóes nacionais ligadas à Metrologia, porém ainda estão sujeitas a revisōes e sugestôes.

É importante ressaltar que também devem ser tomados cuidados com os eletrodos utilizados para a medição de $\mathrm{pH}$. A sua inadequada manutenção pode favorecer a má qualidade de medições. Utilizar medidores e eletrodos não adequados para uso, invalidaria todos os resultados. A estimativa da incerteza das medições assim obtidas não teria sentido nenhum.

Apesar da importância de manter procedimentos de limpeza e manutenção dos eletrodos usados na rotina, os fabricantes dizem pouco a respeito de como fazê-lo e qual sua vida útil. Com referência a instruções em normas técnicas internacionais (BSI, 1984; DIN, 1989, 1985), são sugeridos testes de desempenho do eletrodo. O potencial de referência, a resistência de junção, o potencial da junção e a polaridade são itens que devem ser verificados. Porém, os procedimentos para testes de desempenho e os critérios para a sua aceitação ainda não foram estabelecidos, deixando ressaltada a necessidade da integração de laboratórios para a elaboração das normas técnicas nacionais (Chui et al, 2000).

Enfim, leis e regulamentaçôes para proteção dos recursos hídricos estão bem definidas. Empresas em geral preocupam-se em atingir as metas da NBR/ISO 14000, porém nem todas dão a devida atenção à confiabilidade metrológica de suas mediçôes, bem como os próprios auditores não o fazem em situaçōes de avaliação. A disseminação dos conceitos de rastreabilidade e incertezas em mediçóes só vem a favorecer a qualidade em todos os aspectos da vida humana.

\section{REFERÊNCIAS}

ABNT/ASSOCIAÇÃO BRASILEIRA DE NORMAS TÉCNICAS. Sistemas de Gestão da Qualidade-Requisitos. NBR ISO 9001:2000.

ABNT/ASSOCIAÇĀO BRASILEIRA DE NORMAS TÉCNICAS. Sistemas de Gestão Ambiental. NBR ISO 14000:1996.

BAUCKE, F.G.K., SPITZER, P., NAUMANN, R. Analytical Chemistry News \& Features, v.1, p. 226 A, Apr. 1998.

BIPM/BUREAU INTERNATIONAL DES POIDS ET MESURES. Organisation Intergouvernementall de la Convention du Metre. The International System of Units - SI. 7th Edition, 1998.

BSI/BRITISH STANDARD INSTITUTION, pH Measurement - Specification for reference value standard solutions and operational reference standard solution. BS 1647: Part 2: 1984.

BUCK R.P. et al. Measurement of $p H$. Definition, Standards and Procedures, Pure Appl. Chem., v. 74, p. 2169-2200, 2002.

CHUI, Q.S.H. et al. Medições de $\mathrm{pH}$ nos laboratórios da USF: uma experiência que motivou a implementação de ações para a garantia da qualidade no Laboratório de Farmácia Industrial. In: METROCHEM-2000 - II INTER-AMERICAN CONGRESS ON METROLOGY IN CHEMISTRY, Dec. 4-7, São Paulo, SP, Brasil, p.460-469, 2000.

CNI/CONFEDERAÇÃO NACIONAL DAS INDÚSTRIAS, Estrutura Internacional da $\mathrm{Me}$ trologia Cientifica. www.normalizacao.cni.org. br/metrologia - estrutura_inter.htm, acesso em 30/08/2005

COVINGTON, A.K., BATES, R.G., DURST, R.A. Pure \& Appl.Chemistry, v. 57, n. 3, p. $531-542,1985$.

DECRETO ESTADUAL no 8468, 1979 regulamento da Lei n0 997/76, artigo 18 e 19, sobre prevenção e controle da poluição do meio ambiente no Estado de São Paulo, 1979.

DIN/DEUTSCH I. NORMALIZATION. Glass Electrodes for $p H$ measurement. DIN 19263, 1989.

DIN/DEUTSCH I. NORMALIZATION. Reference Electrodes/pH measurement. DIN 19264, 1985. 
FRAGA, I.C.S. et al. Confiabilidade Metrológica de algumas soluçōes tampão utilizadas para a medição de $p H$. In: ENQUALAB 2002 - ENCONTRO PARA A QUALIDADE DE LABORATÓRIOS, 30/07 a 01/08/2002, Escola SENAI Suiço Brasileira, São Paulo, SP, promoção da REMESP, SENAI e SBM, 2002.

HARRY, F.M., WALLER, R.A. Bayesian $\mathrm{Me}$ thods, in Statistical Methods for Physical Science. New York. J.L. Stanford and S.B. Vaderman, Eds., 1994.

INMETRO/INSTITUTO NACIONAL DE METROLOGIA E QUALIDADE INDUSTRIAL. Vocabulário Internacional de Termos Fundamentais e Gerais em Metrologia-VIM. Rio de Janeiro. Publicação INMETRO (versão traduzida do original em inglês), 1995.

ISO GUM - ISO/INTERNATIONAL ORGANIZATION FOR STANDARDIZATION. Guide to the Expression of Uncertainty in Measurement, Geneva, 1998.
KOCH, W. F. Analytical Chemistry News \& Features, v. 1, p. 700A, Dec. 1997.

OLIVEIRA, E. C. Qualificação da exatidão de um medidor de $p H$ pelas suas fontes de incerteza. In: METROLOGIA 2003 - METROLOGIA PARA A VIDA, 01 a 05/09/2003, Recife, Pernambuco, promovido pela Sociedade Brasileira de Metrologia (SBM), 2003.

SOUZA, V. et al. Implantação do sistema primário de medição de $p H$ na Divisão de Metrologia Química do INMETRO. In: METROLOGIA 2003 - METROLOGIA PARA A VIDA, 01 a 05/09/ 2003, Recife, Pernambuco, Brasil, Sociedade Brasileira de Metrologia (SBM), 2003.

VUOLO, J.H. Avaliação e Expressão de Incertezo de Medição. Revista Brasileira de Ensino de Física, v. 21, n. 3, p. 350-358, Set. 1999.
Endereço para correspondência:

Queenie Siu Hang Chui

Programa de Pós-Graduação em

Engenharia e Ciência dos Materiais -

PPGECM

Rua Alexandre Rodrigues Barbosa, 45

Itatiba

| 325 |-040 São Paulo - SP - Brasil

Tel.: (I I) 45348025

E-mail:queenie.hang@saofrancisco.edu.br

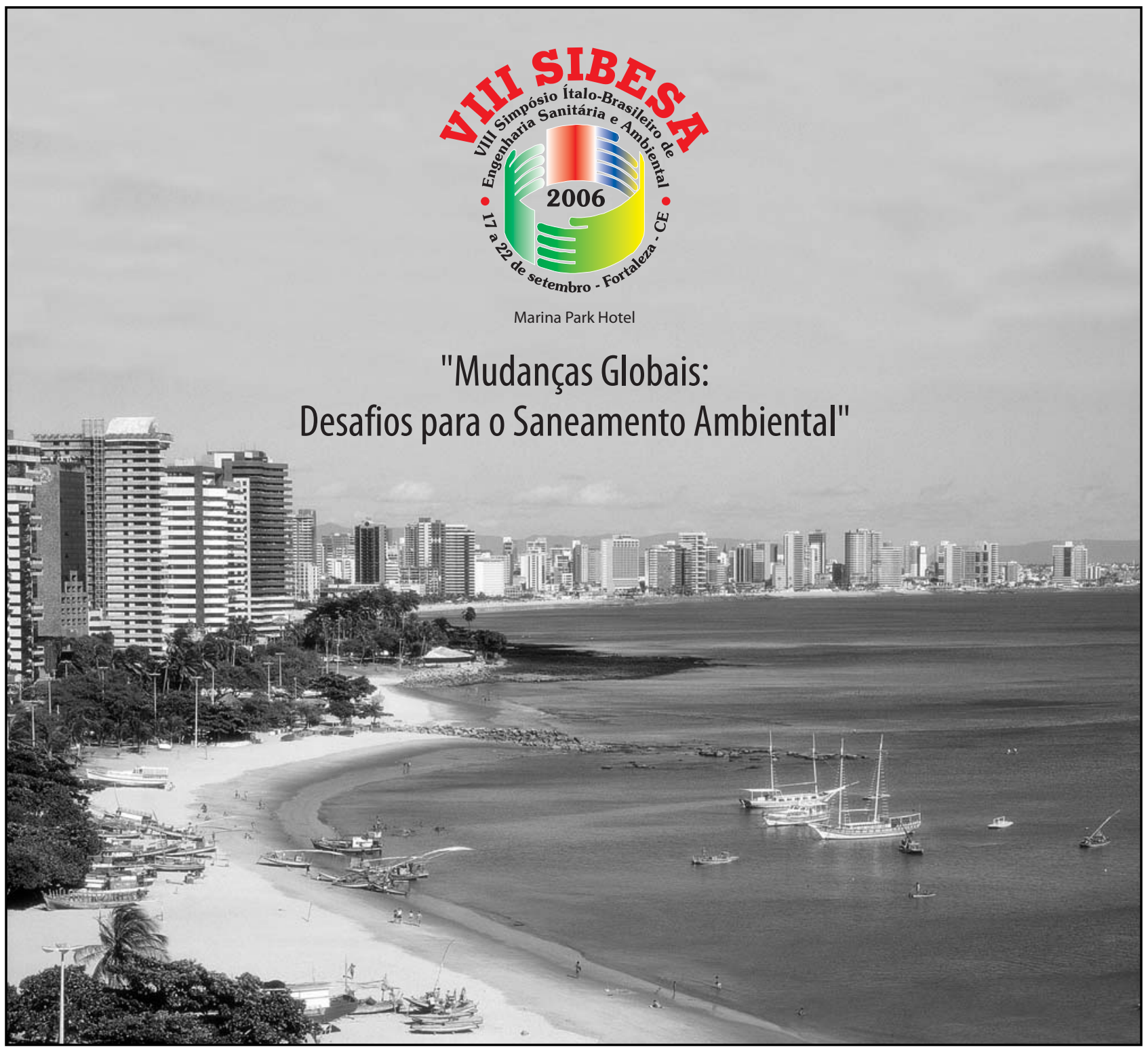

